\title{
Entlassmanagement der Kliniken: Was auf Hausärzte zukommt
}

\begin{abstract}
Der Gemeinsame Bundesausschuss (G-BA) hat am 17. Dezember 2015 die Neuregelungen im $₫ 39$ Abs. 1a SGB V zum sogenannten Entlassmanagement durch Krankenhäuser konkretisiert. Dabei passte er mehrere Richtlinien an, die für die hausärztliche Praxis von Bedeutung sind. Der Gesetzgeber will mit diesen Neuregelungen die Überleitung der Patienten in die vertragsärztliche Versorgung verbessern. Die Beschlüsse liegen noch zur Prüfung beim Bundesgesundheitsministerium.
\end{abstract}

Arzneimittel dürfen Krankenhäuser künftig mit dem kleinsten Packungsgrößenkennzeichen verordnen. Der G-BA hat die Arzneimittelrichtlinien entsprechend angepasst. Das Krankenhaus muss den weiterbehandelnden Arzt rechtzeitig über die medikamentöse Therapie zum Zeitpunkt der Entlassung, die Dosierung und die im Rahmen des Entlassmanagements verordneten Arzneimittel informieren, ggf. inklusive Hinweisen zur Therapiedauer. Wenn das

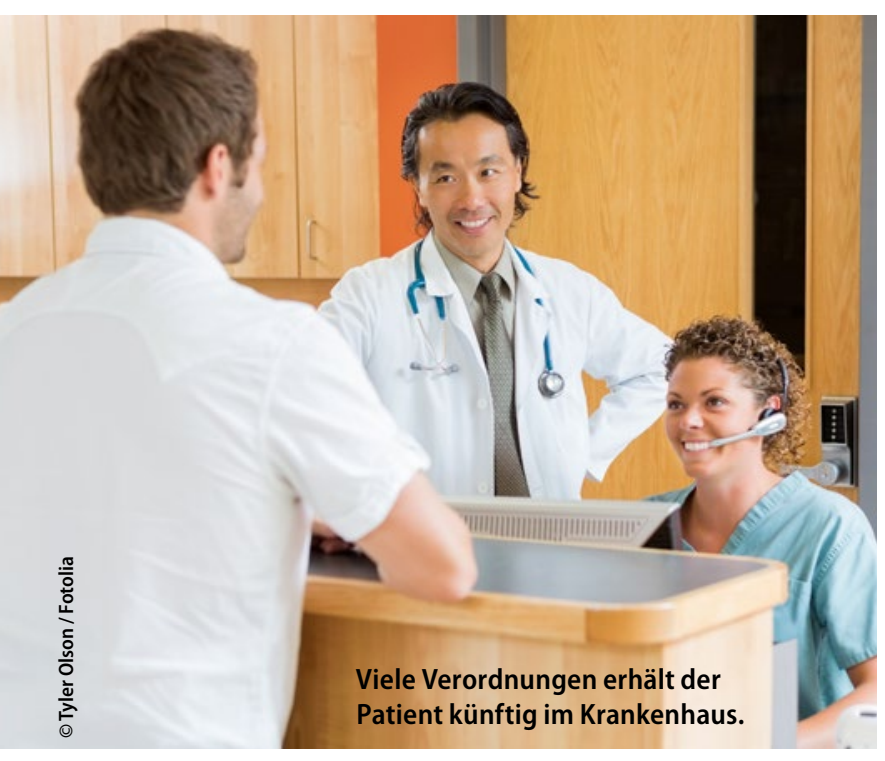

Krankenhaus eine vor Aufnahme bestehende Medikation verändert, muss das dargestellt und erläutert werden.

Das Krankenhaus muss genau prüfen, ob eine Verordnung erforderlich ist. Ein typisches Beispiel ist die Entlassung vor oder am Wochenende, wenn man dem Patienten die Arzneimittel ggf. gemäß $\$ 14$ Abs. 7 Apothekengesetz direkt mitgeben kann. Krankenhausärzte dürfen nicht mehr als eine N1-Packung bzw. die nächstkleinere im Handel befindliche Menge verordnen.

Heilmittel dürfen Krankenhäuser nun auch über einen Verordnungszeitraum von bis zu sieben Kalendertagen nach Entlassung verordnen. Das Entlassungsdatum muss dabei vermerkt sein. Bestehende vertragsärztliche Verordnungen muss der Krankenhausarzt nicht berücksichtigen, und auch der Vertragsarzt muss später bei seinen Verordnungen nicht auf die der Klinik achten.

Hilfsmittel, die zum Verbrauch bestimmt sind (z. B. Inkontinenzartikel), muss das Krankenhaus grundsätzlich so verordnen, dass ein Versorgungszeitraum von bis zu sieben Kalendertagen nach Entlassung gewährleistet ist. Nur wenn diese Menge nicht erhältlich ist, kann die nächstgrößere Einheit verordnet werden.

Braucht der Patient Hilfsmittel, die nicht zum Verbrauch bestimmt sind (z. B. Gehhilfe, Pflegebett), ist die Verordnungsdauer nicht begrenzt. Dauerhaft verwendete Hilfsmittel, die nach der Entlassung individuell angefertigt und dann ärzt-

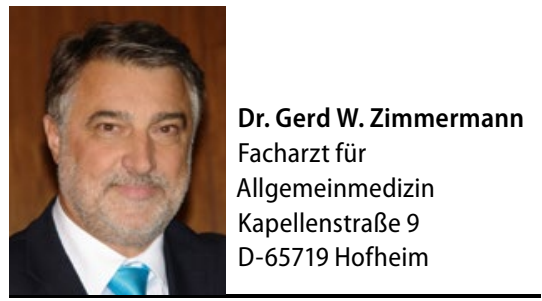

lich kontrolliert werden müssen (z. B. Hör- und Sehhilfen oder Prothesen), sollten in der Regel nicht vom Krankenhaus verordnet werden. Ausnahmen sind möglich, z. B. wenn bereits in der Klinik ein Beatmungsgerät gebraucht wird.

Häusliche Krankenpflege konnten Krankenhausärzte bereits verordnen, bisher für fünf Arbeits- bzw. Werktage nach der Entlassung. Nun werden es sieben Kalendertage.

Bescheinigungen der Arbeitsunfähigkeit können Krankenhausärzte künftig für einen Zeitraum von bis zu sieben Kalendertagen ausstellen.

\section{MMW-KOMMENTAR}

Arzneimittelrezepte im Rahmen des Entlassmanagements müssen als solche gekennzeichnet und binnen drei Werktagen (einschließlich Samstag) eingelöst werden. Das Krankenhaus kann dabei auch verschreibungspflichtige Betäubungsmittel verordnen. Eine vom Krankenhaus verordnete Heilmittelbehandlung muss der Patient innerhalb von sieben Kalendertagen nach Entlassung aufnehmen und innerhalb von zwölf Tagen abschließen. Die restlichen Einheiten verfallen - sodass das Heilmittelbudget des Hausarztes unnötig belastet wird.

Tatsächlich haben diese Neuerungen für den Hausarzt eine große Bedeutung bei der Wirtschaftlichkeitsprüfung. Nur wenn die Krankenhäuser den neuen gesetzlichen Rahmen tatsächlich ausfüllen und Verordnungen nicht weiterhin auf die Niedergelassenen abwälzen, können ungerechtfertigte Prüfungen im Arznei- und Heilmittelbereich verhindert werden. Die Krankenhäuser sind deshalb ausdrücklich verpflichtet, den weiterbehandelnden Vertragsarzt stets über alle Verordnungen zu informieren. Übrigens gelten die Vorgaben grundsätzlich auch für Einrichtungen der medizinischen Rehabilitation. 